\title{
Management Strategies for Patients After CABG Surgery in the Outbreak of the COVID-19 Pandemic
}

This article was published in the following Dove Press journal:

Risk Management and Healthcare Policy

\author{
Xiaogang Wang (iD \\ Yangzhao Zhou ${ }^{1,2}$ \\ Xia Zhang' \\ Jianming $\mathrm{Li}^{\prime}$ \\ Xinmin Zhou' \\ ZhiWei Zhang ${ }^{1,2}$
}

'Department of Cardiovascular Surgery, The Second Xiangya Hospital, Central South University, Changsha, Hunan 4 I00II, People's Republic of China; ${ }^{2}$ Department of Medicine, University of California San Diego, La Jolla, CA 92093, USA
Correspondence: ZhiWei Zhang Department of Cardiovascular Surgery, The Second Xiangya Hospital, Central South University, Changsha, Hunan 41001 I, People's Republic of China Tel $+86 \quad 13787083210$

Email somebody_zhang@csu.edu.cn
Background: The novel coronavirus 2019 pandemic (COVID-19) has quickly spread over the world and affected over 100 countries so far. Patients with pre-existing cardiovascular disease may have a higher risk of infection of COVID-19 and worse outcomes than others. To improve the outcome during the pandemic, management strategies for the patients recovering from coronary artery bypass graft $(\mathrm{CABG})$ surgery need to be reconsidered.

Methods: Some precaution advices including self-protection, blood glucose and blood pressure controlling are recommended for the patients recovering from CABG during the pandemic. They are encouraged to communicate with doctors by telephone or Internet when COVID-19 related symptoms such as cough, fever and dyspnea occur. As a follow-up strategy for patients after CABG surgery, cardiac biomarkers and CTA could also be helpful to the diagnosis of COVID-19. Some medications being investigated for COVID-19 therapy may have side effects relevant to cardiovascular disease. Appropriate personal protection equipment (PPE) is necessary for cardiovascular health-care workers operating in clinical settings.

Results: There was zero out of over 300 follow-up patients after CABG surgery confirmed to be infected with COVID-19 from January to June 2020. No cardiovascular health-care workers were reported to be infected neither in the Second Xiangya Hospital during the pandemic.

Conclusion: The management strategy here we proposed could improve the outcome of patients after $\mathrm{CABG}$ during the pandemic and benefit both cardiovascular patients and health-care workers.

Keywords: cardiovascular patients, CABG, COVID-19 pandemic

\section{Introduction}

First time in December 2019 at Wuhan China, a pneumonia caused by a novel coronavirus was identified. It was found to have very high infectiousness and lethality in human beings. ${ }^{1}$ The epidemic then spread quickly over most of China. Over 4000 confirmed cases were reported from more than 30 provinces in China before the end of January 2020, among which there were more than 900 severe cases and 100 deaths. $^{2}$ To date, the pandemic has affected over 100 countries worldwide and becoming a global public health problem.

The illness is caused by a coronavirus called SARS-CoV-2 (severe acute respiratory syndrome coronavirus 2 ). Some infected patients present severe acute respiratory syndrome consisting of fever, cough, and dyspnea. It is estimated that over $80 \%$ of confirmed infected patients were asymptomatic in the early stage or even during the whole period of infection, which remains as the first challenge of 
the diagnosis and prevention of transmission of novel coronavirus 2019 (SARS-CoV-2). ${ }^{3}$ A diagnostic guideline recommending a combination of chest computed tomography (CT), Transcription-Polymerase Chain Reaction (RTPCR) of coronavirus RNA, and acute respiratory syndrome was published by the Chinese Center for Disease Control and Prevention after the outbreak. The disease dramatically increased the burden on China's hospitals and on inpatient screening, which included patients seeking coronary artery bypass graft (CABG) surgery.

As a therapeutic strategy of myocardial revascularization, CABG surgery can greatly relieve the symptom of chest pain in patients with coronary artery disease (CAD) underlying acute myocardial infarction (AMI). Meanwhile, it has been reported that patients with pre-existing cardiovascular diseases (CVD) have a higher risk of infection of COVID-19 and worse outcomes after infection than other patients. To improve the outcome during the pandemic, management strategies for the patients recovering from $\mathrm{CABG}$ need to be reconsidered. We here propose some ideas regarding this group of patients. From January to June 2020, we gave our recommendations during the pandemic to more than 300 follow-up patients within one-year after CABG. The results indicated that all the cardiovascular events occurred to the patients during the pandemic were handled properly, and that no patient or related cardiovascular health-care worker was infected with COVID-19.

The study was approved by the Ethics Committee of the Second Xiangya Hospital, Central South University in China. All research studies on humans have been performed in accordance with the principles stated in the Declaration of Helsinki. Written informed consent was obtained from all study participants or their legal representatives or guardians.

\section{Methods and Results}

\section{Precautionary Advice for the Patients Recovering from CABG During the Pandemic}

According to the data reported by the Chinese Center for Disease Control and Prevention, elderly patients with preexisting medical comorbidities are prone to severe illness and death after infection, which places the patients recovering from CABG surgery at a higher of infection. ${ }^{2}$ To prevent COVID-19 infection, they carefully protect their health, maintain their indoor environmental hygiene and air circulation, and reduce time spent in crowded public places to the greatest extent possible. These patients should continue wearing face masks, protective eyewear, and gloves when going out. Chinese Center for Disease Control and Prevention has several recommendations to reduce the risk of infection: i) wash the hands with soap and water or with an alcohol-based hand sanitizer; ii) cover the nose and mouth with a tissue when coughing or sneezing or sneeze into your elbow; iii) avoid close contact with anyone who has cold or flu-like symptoms; iv) cook eggs and meats thoroughly; v) avoid unprotected contact with wild or farmed animals. ${ }^{4}$

Diabetes mellitus is present in $25-30 \%$ of patients admitted with the acute coronary syndrome (ACS) and in up to $40 \%$ of patients undergoing $\mathrm{CABG} .{ }^{5,6}$ Some patients recovering from $\mathrm{CABG}$ have pre-existing medical comorbidities including hypertension and hyperlipidemia. It is reported that in the over 40,000 confirmed cases, the casefatality rate of patients with CVD, diabetes, or hypertension is notably higher than average, which indicates the control of blood glucose (BG) and blood pressure (BP) playing a significant role in the prevention and outcome of COVID-19 infection for patients after CABG. ${ }^{3}$ Recommendations place the target BP below 140/90 $\mathrm{mmHg}$, and that of glycosylated hemoglobin below $7 \%{ }^{7-9}$ For patients with both diabetes and hypertension, the recommend target BP is below $130 / 80 \mathrm{mmHg} .{ }^{10}$

Traditionally, CAD patients are older individuals with diabetes, hypertension, or hyperlipidemia. Once infected with COVID-19, they have a higher chance to develop severe cases that present as pneumonia, the acute respiratory distress syndrome, with or without cardiogenic shock. Therefore, patients recovering from CABG surgery need to be especially careful to observe such symptoms as cough, fever, diarrhea, dyspnea, fatigue, and myalgia. It is recommended such patients seek care at an Emergency Department (ED) as soon as any of these symptoms appear. Furthermore, on-pump CABG patients are more likely to contract COVID-19 after surgery than those with off-pump as there is some immunosuppression within 7 days after cardiopulmonary bypass (CPB). More attention should be paid to personal protection after $\mathrm{CPB}$ operation. We also recommend that close contacts of these patients receive the COVID-19 screening test if it available. ${ }^{11}$

\section{In-Home Care Advice for Patients Recovering from CABG}

It is recommended that the patients who receive $\mathrm{CABG}$ surgery should wait at least 3 months before returning to 
work. For a better outcome, patients are advised to i) quit smoking and drinking; ii) eat diet low in fat, low in salt, and high in protein; iii) control their blood pressure and blood glucose properly if they have hypertension or diabetes; iv) engage in moderate indoor or outdoor activities with good protection. During their recovery period, angina pectoris may still occur or even become worse than before surgery. Though the patients and their families are usually informed of this in advance, patients may still become anxious when it happens. Because the epidemic may affect hospital outpatient service, it can be difficult for the patients and their families come to hospitals to seek advice from doctors promptly, and cardiovascular health-care workers including doctors and nurses are encouraged to follow up on the recovery of patients after CABG surgery by telephone or the Internet. Patients and their families can receive advice with respect to drug use, early diagnosis, and initial treatment of disease in a timely manner. Patients and their families should be vigilant regarding COVID-19 symptoms such as fever, cough, and dyspnea. It is highly recommended that patients go to an Emergency Department directly once any such symptom emerges. A combination of chest $\mathrm{CT}$ and nucleic acid test would be helpful to diagnose the COVID-19 infection.

\section{Postoperative Follow-Up of Patients After CABG During the Pandemic}

The follow-up strategy for patients after CABG consists of imagological examination and laboratory testing. The laboratory test should assess white blood cells, hemoglobin, C-reactive protein (CRP), procalcitonin (PCT), and, most importantly, cardiac biomarkers. Cardiac biomarker levels including cardiac troponin (cTn) and brain natriuretic peptide (BNP)/N-terminal proBNP (NT-proBNP) are significant indicators of the level of recovery from myocardial injury for patients after CABG. This is an important part of the post-CABG follow-up strategy. Patients would be tested for their levels frequently when in hospital and encouraged to take a test one to two weeks after returning home. A cTn level at least one value above the 99th percentile upper reference limit indicates an acute myocardial injury. However, it is reported that some COVID-19 patients also show elevated cTn, especially patients admitted to intensive care unit (ICU), and the level of cTn revealed a positive correlation with COVID19 illness, which indicates an increased level of cTn may be associated with worse COVID-19 outcomes. ${ }^{12}$ Mild elevation of cTn in patients recovering from CABG might be related to pre-existing cardiac disease but may also indicate emerging COVID-19 infection. For patients with elevated cTn, the BNP/NT-proBNP level is also reported to be increased, which may be because of the combination of pre-existing cardiac disease and COVID19 infection.

Computed tomography angiography (CTA) is recommended in patients with prior revascularization because it is non-invasive. Ever since the COVID-19 outbreak began, chest CT has been one of the preferred methods for the clinical screening and diagnosis of COVID-19. For those patients without such symptoms as cough, fever, or dyspnea, some chest CT findings can facilitate the diagnosis of COVID-19. In some hospitals in China, every inpatient is required to undergo a chest $\mathrm{CT}$ for the screening of COVID-19 infection. A combination of CTA and chest CT could be beneficial for the prevention and early diagnosis of COVID-19 in patients after CABG.

\section{Consideration of Drug Use}

Antiretroviral therapy was considered a possible cure for COVID-19 ever since the outbreak began, but some antiretroviral medicines may involve cardiovascular toxicity and require re-consideration before they can be recommended for use in patients recovering from CABG. With a potential side effect of prolonging PR- and QT- interval, ritonavir and lopinavir may be not an appropriate medication for the treatment of COVID-19 to patients after CABG. ${ }^{13}$ As a frontline anticoagulant medicine, clopidogrel and ticagrelor are recommended for the first 6 months after CABG surgery. Their active metabolites and serum concentration may be influenced by the use of ritonavir and lopinavir.

Antiplatelet agents play an important role in preventing ischemic events. Long-term aspirin use (75-100 mg, once per day) is recommended for patients recovering from CABG, while the risk of ischemia and bleeding should be monitored during use.

For the patients recovering from AMI, such betablockers as metoprolol, propranolol, and labetalol have been found to markedly reduce the death rate, by $30 \%$, so it is recommended for use to improve outcomes of patients recovering from CABG. ${ }^{14}$ However, this requires further consideration for cases in which beta-blockers are used in combination with chloroquine, which has been investigated for the prevention of complications of COVID-19. Because of the inhibitive effect of chloroquine 
on CYP2D6, which is metabolized by beta-blockers, careful monitoring of blood pressure and heart rate may be essential for simultaneous use in patients recovering from CABG.

Nitrates are endothelial-dependent vasodilators that can reduce myocardial oxygen demand and improve myocardial perfusion, thereby improving angina symptoms. In the case of angina pectoris attack, nitroglycerin $0.3-0.6 \mathrm{mg}$ may be taken sublingually once every $5 \mathrm{~min}$ until the symptoms are relieved, and the maximum dose must not exceed $1.2 \mathrm{mg}$ in $15 \mathrm{~min}$. Long-acting nitrates are used to reduce the frequency and severity of angina attacks and may increase exercise tolerance.

ACEI drugs can reduce the risk of myocardial infarction, stroke, or other major end events in patients with stable angina pectoris or high-risk coronary heart disease without heart failure. ACEI and ARB can be considered for patients at high risk of hypertension, $\mathrm{LVEF} \leq 40 \%$, diabetes, or chronic kidney disease as long as there are no contraindications.

\section{Advice for Cardiovascular Health Care Workers During the Pandemic}

Because it is a communicable respiratory virus, individuals can contract the infection to each other when exposed to the droplets produced by a COVID-19 patient who has coughed or sneezed.

Appropriate personal protection equipment (PPE) including face masks, eye protection, gloves, and gown is necessary for cardiovascular health-care workers operating in clinical settings. ${ }^{15}$ The sense of self-protection should always be kept in mind for health workers when performing any medical procedure in clinics because over $80 \%$ of infected patients are asymptomatic. Furthermore, some additional PPE, such as air purification respirators, should be considered for use when cardiovascular health-care workers perform cardiopulmonary resuscitation, endotracheal intubation, transesophageal echocardiography, or bag mask ventilation. For patients with cardiac arrest, direct chest compression should be avoided during the pandemic, and external mechanical compression devices should be used instead. Self-monitoring of COVID-19-related symptoms, including cough, fever, and dyspnea, is recommended for cardiovascular health-care workers, and a SARS-CoV-2 virus test should be considered when any kind of suspected exposure to infection occurs.

\section{Discussion}

Since December 2019, the COVID-19 pandemic has spread all over the world and affected many countries with large populations, including China, Italy, Japan, and the United States. The first challenge faced by nearly all the affected countries or areas is the shortage of PPE for both public and health-care workers. Regarding this, a classification management system for people visiting CVD patients might be a solution. ${ }^{16}$ For lower-risk patients, telemedicine could be suitable as this would reduce the chance of direct contact between health-care workers and patients and reduce the risk of infection. For the patients with high risk, appropriate PPE should be essential for both patients and healthcare workers during their visits to the hospital. A rational management strategy for patients recovering from $\mathrm{CABG}$ could be beneficial for both health-care workers and patients.

\section{Ethics Statement}

Subjects have given their written informed consent for the case details to be published. The study was approved by the Ethics Committee of the Second Xiangya Hospital, Central South University in China. All research studies on humans have been performed in accordance with the principles stated in the Declaration of Helsinki. Written informed consent was obtained from all study participants or their legal representatives or guardians.

\section{Acknowledgments}

We would like to thank the patients and their families for participating in this study.

\section{Funding}

This work was supported by the National Natural Science Foundation of China (Grant Nos. 81500187, 81502710).

\section{Disclosure}

The authors have no conflicts of interest to declare for this work.

\section{References}

1. Wang C, Horby PW, Hayden FG, Gao GF. A novel coronavirus outbreak of global health concern. Lancet. 2020;395(10223):470-473. doi:10.1016/S0140-6736(20)30185-9

2. Chinese Center of Disease Control and Prevention. Epidemic update and risk assessment of 2019 novel coronavirus; 2020. 
3. Wu Z, McGoogan JM. Characteristics of and important lessons from the coronavirus disease 2019 (COVID-19) outbreak in China: summary of a report of 72314 cases from the Chinese center for disease control and prevention. JAMA. 2020;323(13):1239. doi:10.1001/ jama.2020.2648

4. Chinese Center of Disease Control and Prevention. Available from: http://www.chinacdc.cn/jkzt/crb/zl/szkb_11803/. Accessed July 1, 2020.

5. Luscher TF, Creager MA, Beckman JA, Cosentino F. Diabetes and vascular disease: pathophysiology, clinical consequences, and medical therapy: part II. Circulation. 2003;108(13):1655-1661. doi:10.1161/01.CIR.0000089189.70578.E2

6. Sousa-Uva M, Neumann FJ, Ahlsson A, et al. 2018 ESC/EACTS guidelines on myocardial revascularization. Eur J Cardiothorac Surg. 2019;55:4-90.

7. Officers A, Coordinators for the ACRGTA, Lipid-Lowering Treatment to Prevent Heart Attack T. Major outcomes in high-risk hypertensive patients randomized to angiotensin-converting enzyme inhibitor or calcium channel blocker vs diuretic: the Antihypertensive and Lipid-Lowering Treatment to Prevent Heart Attack Trial (ALLHAT). JAMA. 2002;288(23):2981-2997. doi:10.1001/jama. 288.23.2981

8. UK Prospective Diabetes Study (UKPDS) Group. Intensive blood-glucose control with sulphonylureas or insulin compared with conventional treatment and risk of complications in patients with type 2 diabetes (UKPDS 33). Lancet. 1998;352(9131):837-853. doi:10. 1016/S0140-6736(98)07019-6

9. Group AC, Patel A, MacMahon S, et al. Intensive blood glucose control and vascular outcomes in patients with type 2 diabetes. $N$ Engl J Med. 2008;358:2560-2572.
10. Turnbull F, Neal B, Algert C, et al. Effects of different blood pressure-lowering regimens on major cardiovascular events in individuals with and without diabetes mellitus: results of prospectively designed overviews of randomized trials. Arch Intern Med. 2005;165:1410-1419.

11. Mayumi H, Zhang QW, Nakashima A, et al. Synergistic immunosuppression caused by high-dose methylprednisolone and cardiopulmonary bypass. Ann Thorac Surg. 1997;63:129-137.

12. Zhou F, Yu T, Du R, et al. Clinical course and risk factors for mortality of adult inpatients with COVID-19 in Wuhan, China: a retrospective cohort study. Lancet. 2020;395(10229):1054-1062. doi:10.1016/S0140-6736(20)30566-3

13. Driggin E, Madhavan MV, Bikdeli B, et al. Cardiovascular considerations for patients, health care workers, and health systems during the COVID-19 pandemic. J Am Coll Cardiol. 2020;75 (18):2352-2371. doi:10.1016/j.jacc.2020.03.031

14. Kernis SJ, Harjai KJ, Stone GW, et al. Does beta-blocker therapy improve clinical outcomes of acute myocardial infarction after successful primary angioplasty? J Am Coll Cardiol. 2004;43 (10):1773-1779. doi:10.1016/j.jacc.2003.09.071

15. Mavioglu HL, Unal EU, Askin G, Kucuker SA, Ozatik MA. Perioperative planning for cardiovascular operations in the COVID-19 pandemic. Turk Gogus Kalp Damar Cerrahisi Derg. 2019;28(2):236-243. doi:10.5606/tgkdc.dergisi.2020.09294

16. Patel V, Jimenez E, Cornwell L, et al. Cardiac surgery during the coronavirus disease 2019 pandemic: perioperative considerations and triage recommendations. J Am Heart Assoc. 2020;9(13):e017042. doi:10.1161/JAHA.120.017042
Risk Management and Healthcare Policy

\section{Publish your work in this journal}

Risk Management and Healthcare Policy is an international, peerreviewed, open access journal focusing on all aspects of public health, policy, and preventative measures to promote good health and improve morbidity and mortality in the population. The journal welcomes submitted papers covering original research, basic science, clinical \& epidemiological studies, reviews and evaluations, guidelines, expert opinion and commentary, case reports and extended reports. The manuscript management system is completely online and includes a very quick and fair peer-review system, which is all easy to use. Visit http://www.dovepress.com/testimonials.php to read real quotes from published authors. 